\title{
BOUNDS FOR JACOBIAN OF HARMONIC INJECTIVE MAPPINGS IN N-DIMENSIONAL SPACE
}

\author{
VLADIMIR BOŽIN, MIODRAG MATELJEVIĆ
}

Research partially supported by MNTRS, Serbia, Grant No. 174032

\begin{abstract}
Using normal family arguments, we show that the degree of the first nonzero homogenous polynomial in the expansion of $n$ dimensional Euclidean harmonic $K$-quasiconformal mapping around an internal point is odd, and that such a map from the unit ball onto a bounded convex domain, with $K<3^{n-1}$, is co-Lipschitz. Also some generalizations of this result are given, as well as a generalization of Heinz's lemma for harmonic quasiconformal maps in $\mathbb{R}^{n}$ and related results.
\end{abstract}

\section{INTRODUCTION}

In his seminal paper, Olli Martio [12] observed that every quasiconformal harmonic mapping of the unit planar disk onto itself is co-Lipschitz. Later, the subject of quasiconformal harmonic mappings was intensively studied by the participants of the Belgrade Analysis Seminar, see for example [8, 11, 15, 16, 18, 19]. Harmonic quasiconformal maps have found applications in Teichmüller theory, among other things. Recently, V. Marković 10 proved that a quasiconformal map of the sphere $\mathbb{S}^{2}$ admits a harmonic quasi-isometric extension to the hyperbolic space $\mathbb{H}^{3}$, thus confirming the well known Schoen Conjecture in dimension 3. Related questions of bi-Lipschitzity and bounds of Jacobian have been studied in a sequence of papers by Kalaj and Mateljević; see also a recent paper of Iwaniec-Onninen [4. The corresponding results for harmonic maps between surfaces were obtained previously by Jost and Jost-Karcher [5, 6]. In the planar case, the complex harmonic function $h$ on a simply connected planar domain can be written in the form $h=f+\bar{g}$, where $f$ and $g$ are holomorphic, so that $\left|f^{\prime}\right|$ satisfies the minimum principle and Lewy's theorem. There is no appropriate analogy in higher dimensions; if $h$ is a harmonic mapping from a domain in $\mathbb{R}^{n}$ to $\mathbb{R}^{n}$ then $\left\|\partial_{j} h\right\|$ is subharmonic, but it does not satisfy the minimum principle in general. In fact, Lewy's theorem is false in dimensions higer than two (see 23], also 2] pp. 25-27 for Wood's counterexample). In a very special case, gradients of harmonic functions in $\mathbb{R}^{3}$, for which Lewy's theorem is true, that also turn out to map unit ball onto a convex domain, are known to be co-Lipschitz (see Astala-Manojlović [1, Mateljević [18]). However, it seems that in general, one needs a different approach in higher dimensions.

For example, in [7] the following general theorem was proved:

Theorem 1.1. A $K$-quasiconformal harmonic mapping $f$ of the unit $n$ dimensional ball $(n>2)$ onto itself is Euclidean bi-Lipschitz, provided that $f(0)=0$ and that $K<2^{n-1}$, where $n$ is the dimension of the space. 
It is an extension of a similar result for hyperbolic harmonic mappings with respect to the hyperbolic metric (see Tam and Wan, 20, 1998). The proof makes use of Möbius transformations in the space, and of a recent Kalaj's result [9], which states that harmonic quasiconformal self-mappings of the unit ball are Lipschitz continuous.

Among other things, in this paper we prove that the above result holds if $K<$ $3^{n-1}$, and when codomain is only assumed to be convex. A suitable application of normal family argument allows us to take a conceptually simpler approach then in 7].

The proof is based on Theorem 3.1, showing that the degree of the first nonzero homogenous polynomial in the expansion of $n$ dimensional Euclidean harmonic quasiconformal mapping around an internal point is odd. We combine this with a distortion property of quasiconformal maps to prove that for $n$ dimensional Euclidean harmonic quasiconformal mappings with $K_{O}(f)<3^{n-1}$, Jacobian is never zero.

Our approach gives motivation to define Jacobian non-zero closed families (see Definition 4.2) for which a generalization of Heinz's lemma is shown; we also prove bounds for Jacobian from above for arbitrary harmonic quasiconformal maps. Generalization of Heinz's lemma allows us to prove Theorem 4.3. namely that harmonic quasiconformal maps from unit ball onto a convex domain, that are from Jacobian non-zero closed families, are co-Lipschitz. Essentially, we show that if a map is not co-Lipschitz, then one can get a map of the same type, for which Jacobian vanishes at some internal point. Several applications are also given.

The content of the paper is as follows. In section 2 we collect some known definitions and results which we use in the paper. Proofs that Jacobians of quasiconformal harmonic maps cannot vanish when $K_{O}<3^{n-1}$ or in the case of gradients of harmonic functions, and that Taylor expansions of quasiconformal harmonic maps have odd lowest degree are subject of section 3 . In section 4 we prove the generalization of Heniz's lemma and the co-Lipschitz properties described above, and related results.

\section{BaCKGRound AND Auxiliary Results}

Throughout this paper, we will consider maps from domains, i.e. open and connected regions, of $\mathbb{R}^{n}$, usually denoted by $\Omega, \Omega^{\prime}$, to $\mathbb{R}^{n}$. We will use notation $\mathbb{B}^{n}$ for the unit ball in $\mathbb{R}^{n}$, and for $x \in \mathbb{R}^{n}$ its norm will be denoted by $\|x\|$. For $x \in \mathbb{R}^{n}$, $a>0$ and $A \subseteq \mathbb{R}^{n}$, by $d(x, A)$ we will denote the Euclidean distance of point $x$ from the set $A$, by $a A$ the set $\{a y \mid y \in A\}$ and by $x+A$ the set $\{x+y \mid y \in A\}$.

By $J_{f}(x)$ we will denote the Jacobian of $f$ at point $x, \partial_{j} f$ will stand for $\frac{\partial f}{\partial x_{j}}$, and $\partial_{i j}^{2} f$ for $\frac{\partial^{2} f}{\partial x_{i} \partial x_{j}}$, where $x=\left(x_{1}, x_{2}, \ldots, x_{n}\right)$ is the vector argument of $f$.

We will consider Euclidean harmonic maps, also called harmonic maps in this paper, i.e. those with zero Laplacian of each coordinate function. Also, we will deal with quasiconformal maps.

For a domain $\Omega$ in $\mathbb{R}^{n}$, a map $f: \Omega \mapsto \mathbb{R}^{n}$ is quasiconformal if it is a homeomorphism of $\Omega$ to $f(\Omega)$, and if $f$ belongs to Sobolev space $W_{1, l o c}^{n}(\Omega)$ and there exists $K, 1 \leq K<\infty$, such that

$$
\|D f(x)\|^{n} \leq K J_{f}(x) \text { a.e. on } \Omega,
$$


where $\|D f(x)\|$ denotes the operator norm of the Jacobian matrix of $f$ at $x$. The smallest $K$ in (2.1) is called the outer dilatation $K_{O}(f)$. The inner dilatation $K_{I}(f)=K_{O}\left(f^{-1}\right)$, and map $f$ is $K$-quasiconformal if $\max \left(K_{O}, K_{I}\right) \leq K$.

We will need the following proposition concerning a distortion property of quasiconformal mappings (see [22]):

Proposition 2.1. If $g: \mathbb{B}^{n} \mapsto \mathbb{B}^{n}$ is quasiconformal, $g(0)=0$ and $1 / \alpha=$ $K_{I}\left(g^{-1}\right)^{1 /(n-1)}$, then for some $m>0,\|g(x)\| \geq m\|x\|^{1 / \alpha}$.

The next theorem concerns harmonic maps onto a convex domain. For the planar version of Theorem 2.1] cf. [13, 14], also [16, pp. 152-153. The space version was communicated on International Conference on Complex Analysis and Related Topics (Xth Romanian-Finnish Seminar, August 14-19, 2005, Cluj-Napoca, Romania), by Mateljević, cf. also [18]. For convenience of the reader, we repeat the proof.

Theorem 2.1. Suppose that $h$ is an Euclidean harmonic mapping from the unit ball $\mathbb{B}^{n}$ onto a bounded convex domain $D=h\left(\mathbb{B}^{n}\right)$, which contains the ball $h(0)+R_{0} \mathbb{B}^{n}$. Then for any $x \in \mathbb{B}^{n}$

$$
d(h(x), \partial D) \geq(1-\|x\|) R_{0} / 2^{n-1} .
$$

Proof. To every $a \in \partial D$ we associate a nonnegative harmonic function $u=u_{a}$. Since $D$ is convex, for $a \in \partial D$, there is a supporting hyper-plane $\Lambda_{a}$, defined as set of all $y$ for which $\left(y-a, n_{a}\right)=0$, where $n_{a}$ is a unit vector such that $\left(y-a, n_{a}\right) \geq 0$ for every $y \in \bar{D}$.

Define $u(x)=\left(h(x)-a, n_{a}\right)$. Since $n_{a}$ is a unit vector, $u(x) \leq\|h(x)-a\|$. Then $u(0)=\left(h(0)-a, n_{a}\right)=d\left(h(0), \Lambda_{a}\right)$. From geometric interpretation it is clear that $d\left(h(0), \Lambda_{a}\right) \geq R_{0}$.

By Harnack's inequality (cf. [3], p. 29), $c_{n}(1-\|x\|) u(0) \leq u(x)$, where $c_{n}=2^{1-n}$. In particular, $c_{n}(1-\|x\|) R_{0} \leq u(x) \leq\|h(x)-a\|$ for every $a \in \partial D$. Hence, for a fixed $x \in \mathbb{B}^{n}, d(h(x), \partial D)=\inf _{a \in \partial D}\|h(x)-a\| \geq c_{n}(1-\|x\|) R_{0}$ and therefore we obtain the required inequality.

To apply normal family arguments, we need the following results; see Vaisala [21.

Theorem 2.2. Suppose that $\Omega$ is a domain in $\overline{\mathbb{R}^{n}}$, that $K \geq 1$ and that $r>0$. If $\mathcal{F}$ is a family of $K$-quasiconformal mappings of $\Omega$ (not necessarily onto a fixed domain), such that each $f \in \mathcal{F}$ omits two points $a_{f}, b_{f}$ whith spherical distance in $\overline{\mathbb{R}^{n}}$ at least $r$, then $\mathcal{F}$ is a normal family.

Theorem 2.3. Let $\left(f_{j}\right), f_{j}: \Omega \mapsto \overline{\mathbb{R}^{n}}$, be a sequence of $K$-quasiconformal maps, which converges pointwise to a mapping $f: \Omega \mapsto \overline{\mathbb{R}^{n}}$. Then there are three possibilities:

A. $f$ is a homeomorphism and the convergence is uniform on compact sets.

B. $f$ assumes exactly two values, one of which at exactly one point; covergence is not uniform on compact sets in that case.

C. $f$ is constant.

Note that the case B does not happen when we use normal families. 


\section{INTERIOR ZEROS OF THE JACOBIAN}

In this section we prove that a quasiconformal harmonic map cannot have lowest degree polynomials in the Taylor expansion of even degree. Because harmonic functions are real analytic, in the neighborhood of a zero of the Jacobian there is a power expansion in terms of coordinates. The following proposition follows directly from the quasiconformality condition:

Proposition 3.1. Suppose that $h$ is a real analytic quasiconformal mapping from a domain $\Omega \subset \mathbb{R}^{n}$ to $\mathbb{R}^{n}$, such that Jacobian is zero at some point $x_{0}$. Then all the degrees of first non-zero homogenous polynomials in the Taylor expansion of the coordinate functions of $h$ around $x_{0}$ are the same.

Now the following theorem holds:

Theorem 3.1. Suppose that $h$ is an Euclidean harmonic quasiconformal mapping from a domain $\Omega \subset \mathbb{R}^{n}$ to $\mathbb{R}^{n}$, such that Jacobian is zero at $x_{0} \in \Omega$. Then the degree of first non-zero homogenous polynomials in the Taylor expansion of $h$ around $x_{0}$ is odd, and the corresponding homogenous polynomial map, obtained by taking the lowest degree homogenous polynomials in the Taylor expansion of the coordinates, is harmonic and quasiconformal.

Proof. Without loss of generality, by restricting to a ball neighbourhood and a suitable change of variable, we may assume that $x_{0}=0$ and that $\Omega=\mathbb{B}^{n}$. Suppose the contrary, that the lowest degree of the first non-zero homogenous polynomials in the Taylor expansion of the coordinates of $h$ is even, say equal to $2 m$. Then consider the sequence of harmonic quasiconformal maps, $h_{j}: \mathbb{B}^{n} \mapsto \mathbb{R}^{n}, h_{j}(x)=j^{2 m} h(x / j)$. Note that the first non-zero homogenous polynomials in the expansion of all the maps $h_{j}$ are the same as for $h$. Because for $j>1$ derivatives of $h$ are bounded uniformly in $j$ and on $\mathbb{B}^{n}$, from Taylor expansion it follows that all the maps $h_{j}$, for $j>1$, are uniformly bounded on the unit ball. Therefore, $\left\{h_{j} \mid j \in \mathbb{N}\right\}$ is a normal family, and a subsequence of our sequence converges to a harmonic mapping $f$ uniformly on compact sets. By elliptic regularity theory (cf. Hölder and Schauder apriori estimates, 3], pp. 60, 90), all the derivatives of the subsequence will converge to the corresponding derivatives of $f$. It follows that coordinates of $f$ are equal to homogenous polynomials of degree $2 m$, since higher degree homogenous polynomials in the expansions of $h_{j}$ tend to zero. In particular, the limit function $f$ is not constant, and by Theorem 2.3, $f$ is quasiconformal, and hence injective. But the limit map $f$ satisfies $f(-x)=f(x)$, which is a contradiction. Similar procedure in the case of odd lowest degree gives the claimed homogenous polynomial harmonic quasiconformal map.

Next, we will combine this theorem with a distortion property of quasiconformal maps.

Proposition 3.2. Suppose that $h: \Omega \mapsto \mathbb{R}^{n}$ is a harmonic quasiconformal map. If $\partial_{j} h\left(x_{0}\right)=0$ and $\partial_{i j}^{2} h\left(x_{0}\right)=0$ for some $x_{0} \in \Omega$, then $K_{O}(h) \geq 3^{n-1}$.

Proof. Without loss of generality, by restricting to ball neighbourhood of $x_{0}$ whose closure is in the domain, and a change of variable which does not change quasiconformal distortion, we can suppose that $x_{0}=0, h\left(x_{0}\right)=0$, and that, by the Taylor formula, there is $M>0$ such that $\|h(x)\| \leq M\|x\|^{3}$ on $\mathbb{B}^{n}$. Let $g=\left(\left.h\right|_{\mathbb{B}^{n}}\right) / M$ 
If $1 / \alpha=K_{I}\left(g^{-1}\right)^{1 /(n-1)}$, then by Proposition 2.1. $m\|x\|^{1 / \alpha} \leq\|g(x)\| \leq\|x\|^{3}$. Hence $K_{O}^{1 /(n-1)} \geq 3$, and therefore $K_{O} \geq 3^{n-1}$, where $K_{O}(g)=K_{I}\left(g^{-1}\right)$.

Theorem 3.2. Suppose that $h: \Omega \mapsto \mathbb{R}^{n}$ is a harmonic quasiconformal map. If $K_{O}(h)<3^{n-1}$, then its Jacobian has no zeros.

Proof. Contrary suppose that $J_{h}\left(x_{0}\right)=0$ for some $x_{0} \in \Omega$. Since $h$ is quasiconformal, by Theorem 3.1 we find $\partial_{i j}^{2} h\left(x_{0}\right)=0$. Now, by Proposition 3.2, $K_{O}(h) \geq 3^{n-1}$ and this yields a contradiction.

\section{BOUndS FOR THE JACOBIAN}

Heinz's lemma-type results can be obtained for quasiconformal harmonic maps, using normal families. To state our results clearly and in their generality, it is useful to give some definitions.

Definition 4.1. We say that a family $\mathcal{F}$ of maps from domains in $\mathbb{R}^{n}$ to $\mathbb{R}^{n}$ is RHTC-closed if the following holds:

- (Restrictions) If $f: \Omega \mapsto \mathbb{R}^{n}$ is in $\mathcal{F}, \Omega^{\prime} \subset \Omega$ is open, connected and nonempty, then $\left.f\right|_{\Omega^{\prime}} \in \mathcal{F}$.

- (Homothety) If $f: \Omega \mapsto \mathbb{R}^{n}$ is in $\mathcal{F}, a \in \mathbb{R}, a>0$ then $g: \Omega \mapsto \mathbb{R}^{n}$ and $h: a \Omega \mapsto \mathbb{R}^{n}$ are in $\mathcal{F}$, where $g(x)=a f(x)$ and $h(x)=f(x / a)$.

- (Translations) If $f: \Omega \mapsto \mathbb{R}^{n}$ is in $\mathcal{F}, t \in \mathbb{R}^{n}$, then $g: \Omega \mapsto \mathbb{R}^{n}$ and $h: t+\Omega \mapsto \mathbb{R}^{n}$ are in $\mathcal{F}$, where $g(x)=t+f(x)$ and $h(x)=f(x-t)$.

- (Completeness) If $f_{j}: \Omega \mapsto \mathbb{R}^{n}, j \in \mathbb{N}$ are in $\mathcal{F},\left(f_{j}\right)$ converges uniformly on compact sets to $g: \Omega \mapsto \mathbb{R}^{n}$, where $g$ is non-constant, then $g \in \mathcal{F}$.

For instance, families of harmonic maps and of gradients of harmonic functions are RTHC-closed. Also, due to Theorem 2.3 for any given $K \geq 1$, a subfamily of $K$-quasiconformal members of a RTHC-closed family is also RTHC-closed.

Definition 4.2. We say that a family $\mathcal{F}$ of harmonic maps from domains in $\mathbb{R}^{n}$ to $\mathbb{R}^{n}$ is non-zero Jacobian closed, if it is RHTC-closed and Jacobians of all maps in the family have no zeros.

Note that uniform convergence on compact sets in the case of harmonic maps implies convergence of higher order derivatives, via Hölder and Schauder apriori estimates (see [3], pp. 60, 90). This is related to elliptic regularity and holds for more general elliptic operators, and not just Laplacian, so that this method applies in that more general setting too.

Theorem 4.1. For every non-zero Jacobian closed family of $K$-quasiconformal harmonic maps, there is a constant $c>0$, such that if $f: \mathbb{B}^{n} \mapsto \mathbb{R}^{n}$ is from the family, $d\left(0, \partial f\left(\mathbb{B}^{n}\right)\right) \geq 1$ and $f(0)=0$, then

$$
J_{f}(0) \geq c \text {. }
$$

Proof. Suppose the contrary, i.e. that the family contains a sequence $\left(f_{j}\right)$ of maps from the unit ball satisfying $\mathbb{B}^{n} \subseteq f_{j}\left(\mathbb{B}^{n}\right)$ and $f_{j}(0)=0$, such that $J_{f_{j}}(0) \rightarrow 0$ as $j \rightarrow \infty$. Multiplying functions by constants less than 1 if necessary, we may assume, without loss of generality, that the boundary of the image $f_{j}\left(\mathbb{B}^{n}\right)$ always contains a point on the unit spere, and thus use a normal family argument (since infinity and point on the unit sphere are on a fixed spherical distance) to pass to a convergent subsequence. Now note that because of the Gehring distortion property 
(see 21] p. 63, 17]), $f_{j}\left(\frac{1}{2} \mathbb{B}^{n}\right)$ will contain a ball around zero of fixed radius, so the limit cannot degenerate to a constant function. But then the limit, say $g$, is in the family. However, by apriori estimates of elliptic regularity theory, derivatives of $f_{j}$ will also converge to derivatives of $g$, and hence $J_{g}(0)=0$, contradicting the non-zero Jacobian assumption.

Note that the same normal family argument gives upper bound for Jacobian, but for general quasiconformal harmonic maps. Namely, the following theorem holds:

Theorem 4.2. There is a constant $c>0$, depending only on $K$, such that if $f: \mathbb{B}^{n} \mapsto \mathbb{R}^{n}$ is K-quasiconformal harmonic, $d\left(0, \partial f\left(\mathbb{B}^{n}\right)\right) \leq 1$ and $f(0)=0$, then

$$
J_{f}(0) \leq c .
$$

Proof. Proof is essentially the same as for Theorem 4.1 we take a sequence of $K$-quasiconformal harmonic maps $\left(f_{j}\right)$ from the unit ball, such that $J_{f_{j}}(0) \rightarrow \infty$ as $j \rightarrow \infty, f_{j}(0)=0$ and $d\left(0, \partial f_{j}\left(\mathbb{B}^{n}\right)\right)=1$, multiplying by constants now greater than one if necessary. This will provide a subsequence with a limit mapping whose Jacobian at zero is finite, a contradiction.

Applying Theorem 2.1 we get the following result regarding co-Lipschitz condition for maps from ball to a convex domain:

Theorem 4.3. Suppose that $h$ is a harmonic quasiconformal mapping from the unit ball $\mathbb{B}^{n}$ onto a bounded convex domain $D=h\left(\mathbb{B}^{n}\right)$, and that $h$ belongs to a non-zero Jacobian closed family of harmonic maps. Then $h$ is co-Lipschitz on $\mathbb{B}^{n}$.

Proof. Let $x_{0}$ be a point in $\mathbb{B}^{n}$. Define $f: \mathbb{B}^{n} \mapsto \mathbb{R}^{n}$ by

$$
f(x)=\frac{h\left(x_{0}+\left(1-\left\|x_{0}\right\|\right) x\right)-h\left(x_{0}\right)}{d\left(h\left(x_{0}\right), \partial D\right)} .
$$

Applying Theorem 4.1 to $f$, and using the fact that norm of the derivative of $K$ quasiconformal map is bounded from below by a constant times $n$-th root of the Jacobian, and using the uniform estimate $\left(1-\left\|x_{0}\right\|\right) /\left(d\left(h\left(x_{0}\right), \partial D\right)\right) \leq 2^{n-1} / d(h(0), \partial D)$ from Theorem 2.1 we get a uniform bound from below for norm of the derivative of $h$, and hence conclude that map is co-Lipschitz.

A special case of interest we get by applying Theorem 4.3, combining it with Theorem 3.2 .

Theorem 4.4. Suppose $h$ is a harmonic $K$-quasiconformal mapping from the unit ball $\mathbb{B}^{n}$ onto a bounded convex domain $D=h\left(\mathbb{B}^{n}\right)$, with $K<3^{n-1}$. Then $h$ is co-Lipschitz on $\mathbb{B}^{n}$.

\section{REFERENCES}

[1] K. Astala, V. Manojlović, Pavlovic's theorem in space, arXiv:1410.7575 [math.CV]

[2] P. Duren, Harmonic mappings in the plane, Cambridge Univ. Press, 2004.

[3] D. Gilbarg, N. S. Trudinger, Elliptic Partial Differential Equations of Second Order Springer-Verlag Berlin Heidelberg New York Tokyo, 2001.

[4] T. Iwaniec And J. Onninen, Radó-Kneser-Choquet theorem, Bull. London Math. Soc. 46 (2014) 1283-1291

[5] J. Jost, Harmonic Maps Between surfaces, Springer-Verlag Berlin Heidelberg New York Tokyo, 1984.

[6] J. Jost, Two-dimensional Geometric Variational Problems, John Wiley \& Sons, 1991. 
[7] D. Kalaj, M. S. MatelJević, Harmonic quasiconformal self-mappings and Möbius transformations of the unit ball. Pacific journal of mathematics. ISSN 0030-8730. 247 : 2 (2010), 389-406.

[8] D. KalaJ, Harmonic and quasiconformal functions between convex domains, Doctoral Thesis, University of Belgrade, submited 2000, (2002).

[9] D. KalaJ, A priori estimate of gradient of a solution to certain differential inequality and quasiregular mappings, Journal d'Analyse Mathématique, (2013), Volume 119, Issue 1, pp. 63-88.

[10] V. MARKović Harmonic maps between 3-dimensional hyperbolic spaces. Inventiones Mathematicae, DOI 10.1007/s00222-014-0536-x, http://arxiv.org/pdf/1308.1710.pdf

[11] V. Marković, M. Mateljević, A new version of the main inequality and the uniqueness of harmonic maps, Journal d'Analyse Mathématique, (1999), Volume 79, Issue 1, pp. 315-334.

[12] O. Martio, On harmonic quasiconformal mappings, Ann. Acad. Sci. Fenn., A 1, 425, 3-10 (1968)

[13] M. Mateljević, Estimates for the modulus of the derivatives of harmonic univalent mappings, Proceedings of International Conference on Complex Analysis and Related Topics (IX ${ }^{\text {th }}$ Romanian-Finnish Seminar, 2001), Rev Roum Math Pures Appliq (Romanian Journal of Pure and Applied mathematics) 47 (2002) 5-6, 709 -711.

[14] M. Mateljević, Distortion of harmonic functions and harmonic quasiconformal quasiisometry, Revue Roum. Math. Pures Appl. Vol. 51 (2006) 5-6, 711-722.

[15] M. MatelJević, Quasiconformality of harmonic mappings between Jordan domains 2, Filomat 26:3 (2012) 479-509.

[16] M. Mateljević, Topics in Conformal, Quasiconformal and Harmonic maps, Zavod za udžbenike, Beograd 2012.

[17] M. MatelJević, Distortion of quasiregular mappings and equivalent norms on Lipschitz-type spaces, Abstract and Applied Analysis Volume 2014 (2014), Article ID 895074, 20

[18] M. Mateljević, The lower bound for the modulus of the derivatives and Jacobian of harmonic injective mappings http://arxiv.org/pdf/] 1501.03197v2 [math.CV] 7 Feb 2015

[19] M. PAVlović, Boundary correspondence under harmonic quasiconformal homeomorfisms of the unit disc, Ann. Acad. Sci. Fenn., Vol 27, (2002) 365-372.

[20] L. TAM, T. WAN, On quasiconformal harmonic maps, Pacific J Math, V 182 (1998), 359-383

[21] J. VÄISÄLÄ, Lectures on n-Dimensional Quasiconformal Mappings, Springer-Verlag, 1971.

[22] M. Vuorinen, Conformal geometry and quasiregular mappings, Lecture Notes in Math. V 1319, Springer-Verlag, 1988.

[23] J. C. Wood, Lewy's theorem fails in higher dimensions, Math. Scand. 69 (1991), 166.

Faculty of mathematics, Univ. of Belgrade, Studentski Trg 16, Belgrade, Serbia

E-mail address: bozin@matf.bg.ac.rs

E-mail address: miodrag@matf.bg.ac.rs 